\title{
Reduced renal length and volume 20 years after very preterm birth
}

\author{
Mandy G. Keijzer-Veen • Annick S. Devos • \\ Morteza Meradji • Friedo W. Dekker • Jeroen Nauta • \\ Bert J. van der Heijden
}

Received: 28 April 2009 /Revised: 1 October 2009 /Accepted: 2 October 2009 /Published online: 16 December 2009

(C) The Author(s) 2009. This article is published with open access at Springerlink.com

\begin{abstract}
Intrauterine growth retardation is presumed to be associated with decreased renal size and impaired renal function as a result of stunted kidney development and nephron deficit. To study whether very preterm birth also affects renal size at young adulthood, we sonographically measured bipolar kidney length and volume in 51 very premature individuals $(<32$ weeks of gestation), either small (SGA) or appropriate (AGA) for gestational age (22 SGA and 29 AGA), and 30 full-term controls 20 years after birth. Relative kidney length and volume were calculated. Both absolute and relative left kidney length and volume were significantly lower in SGA and AGA individuals, notably in women. Renal size did not differ between SGA and AGA individuals. In $70 \%$ of controls, the left kidney was larger than the right one compared with $40.9 \%$ in SGA [relative risk (RR) 1.7; 95\% confidence interval (CI) 1.0 -3.0] and $48.3 \%$ in AGA (RR 1.5; 95\% CI 0.9-2.3)
\end{abstract}

M. G. Keijzer-Veen · J. Nauta $\cdot$ B. J. van der Heijden Department of Pediatric Nephrology, Erasmus MC - Sophia Children's Hospital, University Medical Center Rotterdam, Rotterdam, The Netherlands

A. S. Devos $\cdot$ M. Meradji

Department of Pediatric Radiology, Erasmus MC - Sophia Children's Hospital, University Medical Center Rotterdam, Rotterdam, The Netherlands

\section{F. W. Dekker}

Department of Clinical Epidemiology,

Leiden University Medical Center,

Leiden, The Netherlands

M. G. Keijzer-Veen $(\square)$

Erasmus MC - Sophia Children's Hospital,

University Medical Center Rotterdam,

Room - SP 2471, PO Box 2060, 3000 CB Rotterdam,

The Netherlands

e-mail: m.keijzer-veen@erasmusmc.nl individuals. Renal structural anomalies were present in eight prematurely born participants only. Our data suggest that kidney growth is stunted after preterm birth, especially on the left side, and in the female gender.

Keywords Renal growth $\cdot$ Kidney development .

Developmental origins of health and disease .

Renal ultrasound Preterm birth .

Intrauterine growth retardation

\section{Introduction}

An increasing number of studies show an association between intrauterine growth retardation (IUGR) and underdevelopment of the fetal kidneys, with limited nephron number and decreased renal size [1-4]. Furthermore, birth weight appears to be a strong determinant of renal size, nephron number, glomerular volume, albuminuria and systolic blood pressure $[5,6]$. These findings add weight to the hypothesis that IUGR carries a risk of renal function loss as a result of nephron deficit, loss of filtration surface area, hyperfiltration, glomerular hypertension, and glomerular damage [7]. As nephrogenesis continues until 36 weeks of gestation, very preterm babies (gestational age $<32$ weeks) are likely to show a nephron deficit at birth [8]. In addition, preterm birth is known to be associated with impaired nephrogenesis $[9,10]$ and limited postnatal kidney growth until the age of 18 24 months [11, 12]. Whether adult renal size is also impaired after premature birth compared with full-term birth or whether renal catch-up growth during childhood is present has not yet been studied.

We report reduced renal size (length and volume) in 20 -year-old individuals born very prematurely [13]. Renal 
size was correlated with glomerular filtration rate (GFR) $\left(\mathrm{ml} / \mathrm{min} / 1.73 \mathrm{~m}^{2}\right)$ and effective renal plasma flow $(\mathrm{ml} / \mathrm{min} /$ $1.73 \mathrm{~m}^{2}$ ). The data reported here documents an additional analysis on renal size in the same cohort.

\section{Methods}

\section{Study population}

We recruited three groups of 20-year-olds: (1) Those born very prematurely $(<32$ weeks) and small for gestational age (SGA), (2) those born very prematurely and appropriate for gestational age (AGA), and (3) those born full term (3742 weeks) and AGA (controls). The SGA and AGA groups participated in a follow-up study of almost all (94\%) of live-born individuals in the Netherlands with a gestational age $<32$ weeks and/or a birth weight $<1,500 \mathrm{~g}$. This is the POPS cohort: Project on Prematures and Small for Gestational Age infants. Participants in the our study had participated in the most recent POPS 19 study $(N=422)$ [14]. Recruitment of SGA individuals started with the lowest birth weight adjusted for gestational age $[\leq-2$ standard deviation scores (SDS) $N=29$ ] and that of AGA individuals with the highest birth weight adjusted for gestational age (0-2 SDS $N=205)$. Controls born between 1 January 1982 and 31 December 1984 were recruited by advertisement posted in the Erasmus Medical Center, Rotterdam, The Netherlands.

\section{Renal ultrasound}

Participants underwent renal function testing, blood pressure measurement, and renal ultrasound study in the Erasmus MC Sophia Children's Hospital, Rotterdam [13]. Each of the three pediatric radiologists performed renal ultrasound by standardized protocol. Renal size was measured in the prone position with a Philips ATL HDI 5000 scanner with a C5-2 curved-array transducer (Philips Nederland BV, Eindhoven, The Netherlands). Measures of maximal bipolar kidney length, width, and thickness were obtained for both kidneys. Renal width and thickness were measured at the level of the kidney hilum. Renal volume ( $\mathrm{ml}$ ) was calculated using the formula for an ellipsoid: $(\pi / 6) *$ length $(\mathrm{cm}) *$ width $(\mathrm{cm}) *$ thickness $(\mathrm{cm})$ [15]. Cortical thickness was defined as the distance between the external surface of the kidney and top of the medulla. As absolute renal size is known to be associated with body size, we calculated the relative kidney length length (bipolar kidney length $(\mathrm{cm})$ / body height $(\mathrm{m})$ ) and relative kidney volume (bipolar kidney length (ml) / body surface area $\left.\left(\mathrm{BSA}, \mathrm{m}^{2}\right)\right)[16]$. Body surface area (BSA) was calculated using the equation $\sqrt{ }($ (body height $(\mathrm{m}) *$ weight $(\mathrm{kg}))$ / 3600). Any renal or ureterovesical anomalies noted were reported.

Intra- and interobserver variability

Intra- and interobserver variability for the three pediatric radiologists were calculated beforehand for measurements

Table 1 Subject baseline characteristics

\begin{tabular}{|c|c|c|c|c|c|c|c|c|}
\hline & \multicolumn{2}{|c|}{ SGA $N=22$} & \multicolumn{2}{|c|}{ AGA $N=29$} & \multicolumn{2}{|c|}{ Controls $N=30$} & \multirow[t]{2}{*}{ ANOVA $p$ value } & \multirow{2}{*}{$\begin{array}{l}\text { Post hoc } p \text { value } \\
\text { Bonferroni }\end{array}$} \\
\hline & Mean & SD & Mean & $\mathrm{SD}$ & Mean & $\mathrm{SD}$ & & \\
\hline Age (years) & 20.7 & 0.2 & 20.7 & 0.4 & 20.7 & 0.8 & 1.0 & \\
\hline Males [\% (number)] & 40.9 & 9 & 37.9 & 11 & 46.7 & 14 & $0.7\left(\chi^{2}\right)$ & \\
\hline BW (g) & 860 & 129 & 1489 & 257 & 3632 & 489 & $<0.001 *$ & All $<0.001$ \\
\hline GA (weeks) & 30.6 & 1.0 & 29.5 & 1.4 & 40.2 & 1.3 & $<0.001 *$ & All $<0.01$ \\
\hline BW (SDS) & -2.3 & 0.3 & 0.7 & 0.5 & 0.3 & 1.0 & $<0.001^{*}$ & $0.09^{\mathrm{a}},<0.001^{\mathrm{b}, \mathrm{c}}$ \\
\hline Body height $(\mathrm{cm})$ & 167 & 11 & 174 & 7.7 & 176 & 10 & $<0.01^{*}$ & $<0.01^{\mathrm{b}}, 0.05^{\mathrm{c}}$ \\
\hline Body height (SDS) & -1.3 & 1.1 & -0.2 & 0.9 & -0.1 & 1.1 & $<0.001 *$ & $<0.001^{\mathrm{b}, \mathrm{c}}$ \\
\hline Body weight (kg) & 60.6 & 9.0 & 67.4 & 12.0 & 71.4 & 11.7 & $<0.01 *$ & $<0.01^{\mathrm{b}}$ \\
\hline Body weight (SDS) & -1.1 & 1.3 & -0.2 & 1.0 & 0.2 & 1.2 & $<0.01^{*}$ & $<0.01^{\mathrm{b}}$ \\
\hline BMI $\left(\mathrm{kg} / \mathrm{m}^{2}\right)$ & 21.7 & 2.6 & 22.1 & 2.8 & 22.9 & 2.8 & 0.2 & \\
\hline BSA & 1.68 & 0.17 & 1.80 & 0.19 & 1.87 & 0.19 & $<0.01^{*}$ & $<0.01^{\mathrm{b}}, 0.05^{\mathrm{c}}$ \\
\hline
\end{tabular}

Data expressed by mean [standard deviation (SD)] and analysis of variance (ANOVA) $p$ value with Bonferroni post hoc multiple testing $S G A$ small for gestational age prematurely born individuals, $A G A$ appropriate for gestational age prematurely born individuals, Controls appropriate for gestational age term-born individuals, $B W$ birth weight, $G A$ gestational age, $B W S D S$ birth weight standard deviation score, $B M I$ body mass index

${ }^{\mathrm{a}}$ AGA vs controls, ${ }^{\mathrm{b}} \mathrm{SGA}$ vs controls, ${ }^{\mathrm{c}} \mathrm{SGA}$ vs AGA

$* p$ value $<0.05$ 
Table 2 Renal size in three study groups

\begin{tabular}{|c|c|c|c|c|c|c|c|c|}
\hline & \multicolumn{2}{|l|}{ Males } & \multicolumn{2}{|c|}{ Females } & \multicolumn{2}{|l|}{ All } & \multirow{2}{*}{$\begin{array}{l}\text { ANOVA } \\
p \text { value }\end{array}$} & \multirow{2}{*}{$\begin{array}{l}\text { Post hoc } p \text { value } \\
\text { Bonferroni }\end{array}$} \\
\hline & Mean & SD & Mean & SD & Mean & SD & & \\
\hline Total absolute kidney length (cm) & & & & & & & $0.007 *$ & $0.007^{\mathrm{b} *}$ \\
\hline $\mathrm{SGA}(N=22)$ & 20.7 & 1.3 & 19.9 & 1.5 & 20.2 & 1.5 & & \\
\hline $\operatorname{AGA}(N=29)$ & 21.3 & 2.0 & 20.3 & 1.3 & 20.7 & 1.6 & & \\
\hline Controls $(N=30)$ & 21.9 & 2.5 & 21.4 & 1.0 & 21.7 & 1.8 & & \\
\hline Total absolute kidney volume (ml) & & & & & & & $0.005^{*}$ & $0.033^{\mathrm{a} *}$ \\
\hline $\operatorname{SGA}(N=22)$ & 298.7 & 76.6 & 216.2 & 58.1 & 249.9 & 76.7 & & $0.008^{\mathrm{b} *}$ \\
\hline $\operatorname{AGA}(N=29)$ & 286.8 & 60.3 & 248.8 & 35.9 & 263.2 & 49.3 & & \\
\hline Controls $(N=30)$ & 313.7 & 93.9 & 307.3 & 67.9 & 310.3 & 79.7 & & \\
\hline Left absolute kidney length (cm) & & & & & & & $0.001 *$ & $0.022^{\mathrm{a} *}$ \\
\hline $\operatorname{SGA}(N=22)$ & 10.1 & 0.6 & 9.9 & 0.7 & 10.0 & 0.7 & & $0.001^{\mathrm{b} *}$ \\
\hline AGA $(N=29)$ & 10.6 & 1.4 & 10.2 & 0.9 & 10.4 & 1.1 & & \\
\hline Controls $(N=30)$ & 11.1 & 1.4 & 11.0 & 0.6 & 11.1 & 1.0 & & \\
\hline Left relative kidney length $(\mathrm{cm} / \mathrm{m})$ & & & & & & & $0.044^{*}$ & $0.059^{\mathrm{a} *}$ \\
\hline SGA $(N=22)$ & 5.7 & 0.3 & 6.2 & 0.4 & 6.0 & 0.4 & & \\
\hline AGA $(N=29)$ & 5.9 & 0.7 & 6.0 & 0.5 & 6.0 & 0.6 & & \\
\hline Controls $(N=30)$ & 6.1 & 0.7 & 6.5 & 0.3 & 6.3 & 0.6 & & \\
\hline Left absolute kidney volume (ml) & & & & & & & $<0.001 *$ & $0.003^{\mathrm{a} *}$ \\
\hline SGA $(N=22)$ & 147.8 & 38.6 & 107.1 & 26.2 & 123.8 & 37.1 & & $0.001^{\mathrm{b} *}$ \\
\hline $\operatorname{AGA}(N=29)$ & 139.0 & 32.4 & 123.0 & 19.4 & 129.1 & 25.8 & & \\
\hline Controls $(N=30)$ & 164.6 & 40.1 & 157.0 & 45.4 & 160.5 & 42.4 & & \\
\hline Left relative kidney volume $\left(\mathrm{ml} / \mathrm{m}^{2}\right)$ & & & & & & & $0.006^{*}$ & $0.008^{\mathrm{a} *}$ \\
\hline SGA $(N=22)$ & 82.9 & 22.5 & 67.0 & 14.2 & 73.5 & 19.3 & & $0.045^{\mathrm{b} *}$ \\
\hline $\operatorname{AGA}(N=29)$ & 70.1 & 12.3 & 72.4 & 10.9 & 71.5 & 11.3 & & \\
\hline Controls $(N=30)$ & 83.3 & 17.1 & 88.8 & 26.6 & 86.2 & 22.4 & & \\
\hline Left cortical thickness $(\mathrm{cm})$ & & & & & & & 0.188 & \\
\hline SGA $(N=22)$ & 1.0 & 0.3 & 0.9 & 0.2 & 0.9 & 0.2 & & \\
\hline $\operatorname{AGA}(N=29)$ & 0.8 & 0.3 & 1.0 & 0.3 & 0.9 & 0.3 & & \\
\hline Controls $(N=30)$ & 1.0 & 0.4 & 1.1 & 0.4 & 1.1 & 0.4 & & \\
\hline Right absolute kidney length (cm) & & & & & & & 0.207 & \\
\hline SGA $(N=22)$ & 10.5 & 0.8 & 10.0 & 0.9 & 10.2 & 0.9 & & \\
\hline $\operatorname{AGA}(N=28)$ & 10.7 & 0.7 & 10.1 & 0.7 & 10.3 & 0.7 & & \\
\hline Controls $(N=30)$ & 10.8 & 1.3 & 10.4 & 0.6 & 10.6 & 1.0 & & \\
\hline Right relative kidney length $(\mathrm{cm} / \mathrm{m})$ & & & & & & & 0.360 & \\
\hline SGA $(N=22)$ & 6.0 & 0.4 & 6.2 & 0.5 & 6.1 & 0.4 & & \\
\hline $\operatorname{AGA}(N=29)$ & 5.9 & 0.2 & 5.9 & 0.5 & 5.9 & 0.4 & & \\
\hline Controls $(N=30)$ & 5.9 & 0.6 & 6.1 & 0.3 & 6.0 & 0.5 & & \\
\hline Right absolute kidney volume (ml) & & & & & & & 0.103 & \\
\hline SGA $(N=22)$ & 150.9 & 44.4 & 109.1 & 34.9 & 126.2 & 43.5 & & \\
\hline $\operatorname{AGA}(N=29)$ & 147.9 & 35.1 & 125.7 & 22.4 & 134.1 & 29.4 & & \\
\hline Controls $(N=30)$ & 149.1 & 57.6 & 150.3 & 37.0 & 149.7 & 46.8 & & \\
\hline Right relative kidney volume $\left(\mathrm{ml} / \mathrm{m}^{2}\right)$ & & & & & & & 0.473 & \\
\hline SGA $(N=22)$ & 84.1 & 24.0 & 68.0 & 19.4 & 74.6 & 22.4 & & \\
\hline $\operatorname{AGA}(N=29)$ & 75.0 & 16.5 & 73.8 & 10.7 & 74.2 & 12.9 & & \\
\hline Controls $(N=30)$ & 74.7 & 25.1 & 84.4 & 18.9 & 79.9 & 22.2 & & \\
\hline
\end{tabular}


Table 2 (continued)

\begin{tabular}{|c|c|c|c|c|c|c|c|c|}
\hline & \multicolumn{2}{|l|}{ Males } & \multicolumn{2}{|c|}{ Females } & \multicolumn{2}{|l|}{ All } & \multirow{2}{*}{$\begin{array}{l}\text { ANOVA } \\
p \text { value }\end{array}$} & \multirow{2}{*}{$\begin{array}{l}\text { Post hoc } p \text { value } \\
\text { Bonferroni }\end{array}$} \\
\hline & Mean & SD & Mean & SD & Mean & SD & & \\
\hline Right cortical thickness $(\mathrm{cm})$ & & & & & & & 0.059 & \\
\hline $\operatorname{SGA}(N=22)$ & 0.8 & 0.2 & 0.8 & 0.3 & 0.8 & 0.2 & & \\
\hline AGA $(N=29)$ & 0.8 & 0.3 & 0.8 & 0.3 & 0.8 & 0.3 & & \\
\hline Controls $(N=30)$ & 1.0 & 0.5 & 1.0 & 0.3 & 1.0 & 0.4 & & \\
\hline
\end{tabular}

Data expressed by mean [standard deviation (SD)] with analysis of variance (ANOVA) $p$ value between groups for all individuals (males and females combined) and Bonferroni post hoc multiple testing between groups

$S G A$ small for gestational age prematurely born individuals, $A G A$ appropriate for gestational age prematurely born individuals, controls appropriate for gestational age term-born individuals

${ }^{\mathrm{a}}$ AGA vs controls, ${ }^{\mathrm{b}} \mathrm{SGA}$ vs controls

${ }^{*} p$ value $<0.05$

in seven healthy students (five females, two males). The mean (95\% limits of agreement) intraobserver variability for bipolar renal length ranged between $0.0 \mathrm{~cm}(-1.1$ to 1.1$)$ and $0.1 \mathrm{~cm}(-1.0$ to 1.2$)$ for the left kidney and $0.1 \mathrm{~cm}$ $(-0.7$ to 0.9$)$ and $0.4 \mathrm{~cm}(-0.5$ to 1.4$)$ for the right kidney. The intraclass coefficient (ICC) for intraobserver variability was 0.97 for left bipolar kidney length and 0.88 for right bipolar kidney length. The mean (95\% limits of agreement) interobserver variability for bipolar renal length ranged between $0.0 \mathrm{~cm}(-1.4$ to 1.5$)$ and $0.2 \mathrm{~cm}(-1.0$ to 1.5$)$ for the left kidney and $0.1 \mathrm{~cm}(-0.1$ to 0.3$)$ and $0.2 \mathrm{~cm}(-0.4$ to 0.8 ) for the right kidney. The ICC for interobserver variability was 0.84 for left bipolar kidney length and

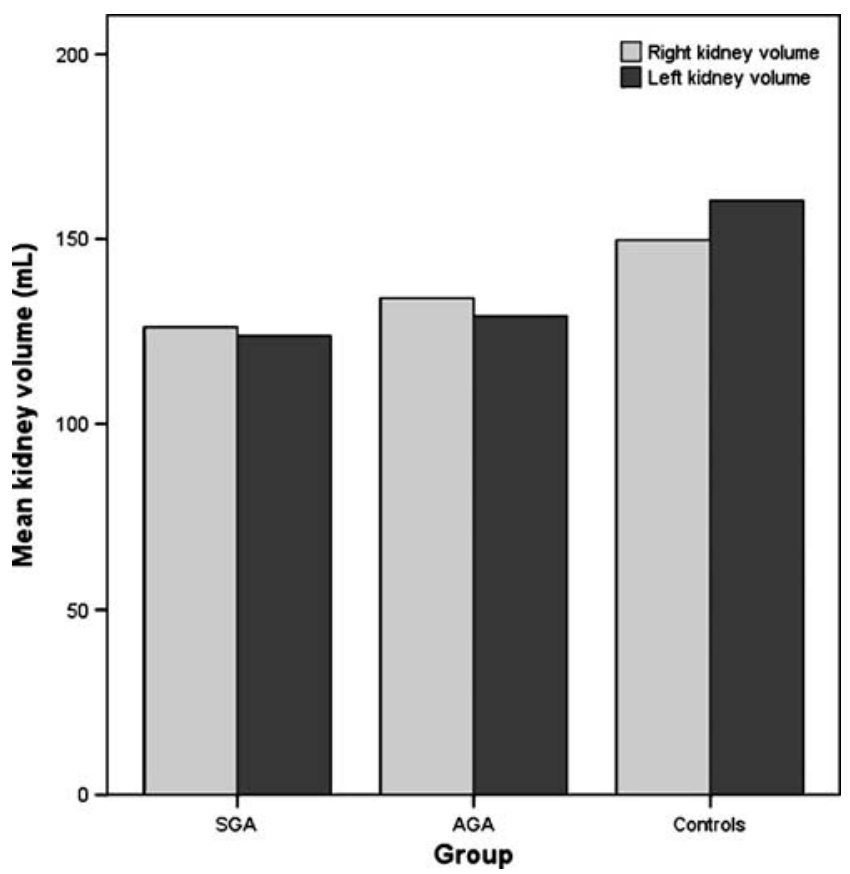

Fig. 1 Mean kidney volume in right and left kidney in SGA, AGA and Controls
0.86 for right bipolar kidney length. Renal volume estimates varied more widely, but significant systematic errors within and between observers did not occur.

Informed consent and ethics committee

Informed consent was obtained from all participants after oral and written information had been given. The Erasmus MC University Medical Center ethical review board approved the study protocol.

\section{Statistics}

Statistical analysis was performed with SPSS 15.0 software. Results are presented as mean and SD. One-way analysis of

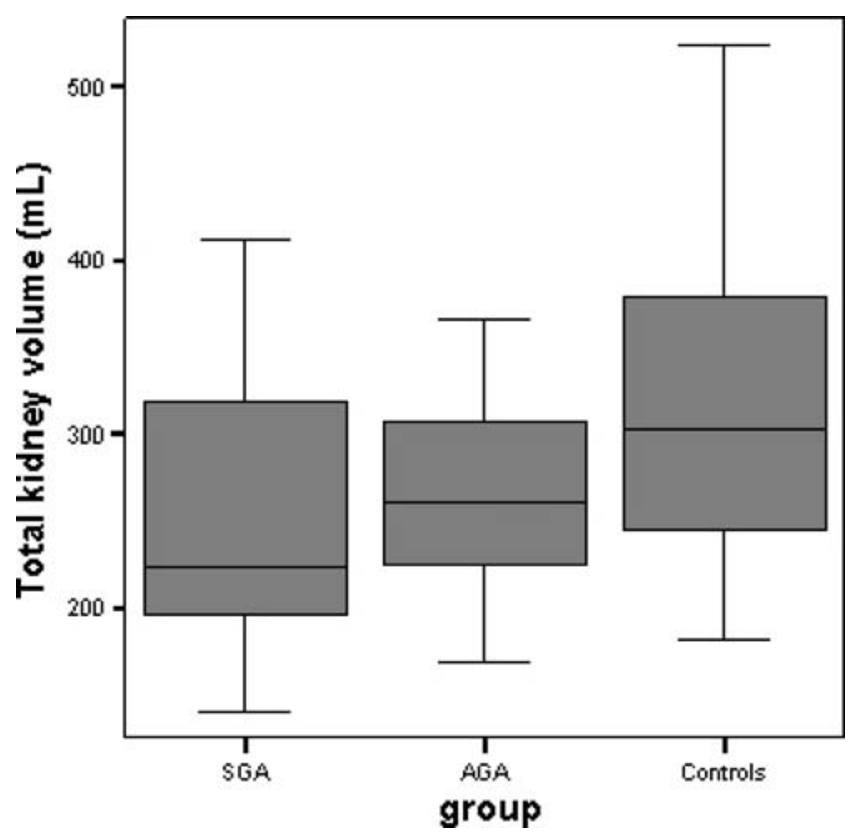

Fig. 2 Total kidney volume in the three study groups 
variance (ANOVA) with post hoc multiple comparisons (Bonferroni) was used to analyze group differences in baseline characteristics. Pearson's chi-square tests were performed to compare proportions of males between study groups; Fisher exact test was used to compare prevalences of renal anomalies. Multivariate regression analysis was used to determine regression coefficients in the association between body height or BSA and renal length and volume. Statistical significance was considered at the level of $5 \%$.

\section{Results}

Baseline characteristics of study population

Eighty-two individuals agreed to participate: i.e. 23 SGA individuals, 29 AGA individuals, and 30 controls. However, one SGA individual suffered an unrelated allergic episode prior to study and did not undergo renal ultrasound, reducing the SGA group to 22. Baseline characteristics are shown in Table 1. Birth weight and gestational age significantly differed between the three groups. Current weight, height, and BSA of SGA individuals were lower than those of AGA individuals and controls, but body mass index (BMI) did not differ between groups.

\section{Renal size}

Renal size is detailed in Table 2. Male individuals had significantly larger kidney volume than female individuals (ANOVA $p$ value $<0.05$ ). Left kidneys were larger than right kidneys in $70 \%$ of controls, in $48.3 \%$ of AGA individuals [relative risk (RR) 1.5 ; $95 \%$ confidence interval (CI) $0.9-2.3$ ], and in $40.9 \%$ of SGA individuals (RR 1.7; 95\% CI 1.0-3.0) (Fig. 1). Total kidney volume in both SGA and AGA individuals was significantly smaller than that in controls $(p<0.05)$ (Table 2, Fig. 2). Also, absolute and relative left renal length and volume in SGA and AGA individuals were smaller than in controls. Right renal size did not differ between groups. Both left and right kidney size did not differ between SGA and AGA individuals. Cortical thickness did not differ between groups. Interestingly, after stratification for sex, all the above differences proved statistically significant in the female gender only.

Male and female individuals in the control group did not differ with regard to renal size for both kidneys. Male and female individuals in both the SGA and AGA groups differed statistically significantly with regard to total kidney volume (ANOVA $p$ value $<0.05$ ). BSA was positively related to volume $\left[\beta 215 \mathrm{ml} / \mathrm{m}^{2}\right.$ (95\% CI 147-283); $p<$ $0.001]$ as shown in Fig. 3a. Body height was positively related to renal length $[\beta 0.09 \mathrm{~cm} / \mathrm{cm}(95 \%$ CI $0.06-0.12)$; $p<0.001]$ as shown in Fig. 3b. In Fig. 4a-d, BSA and body
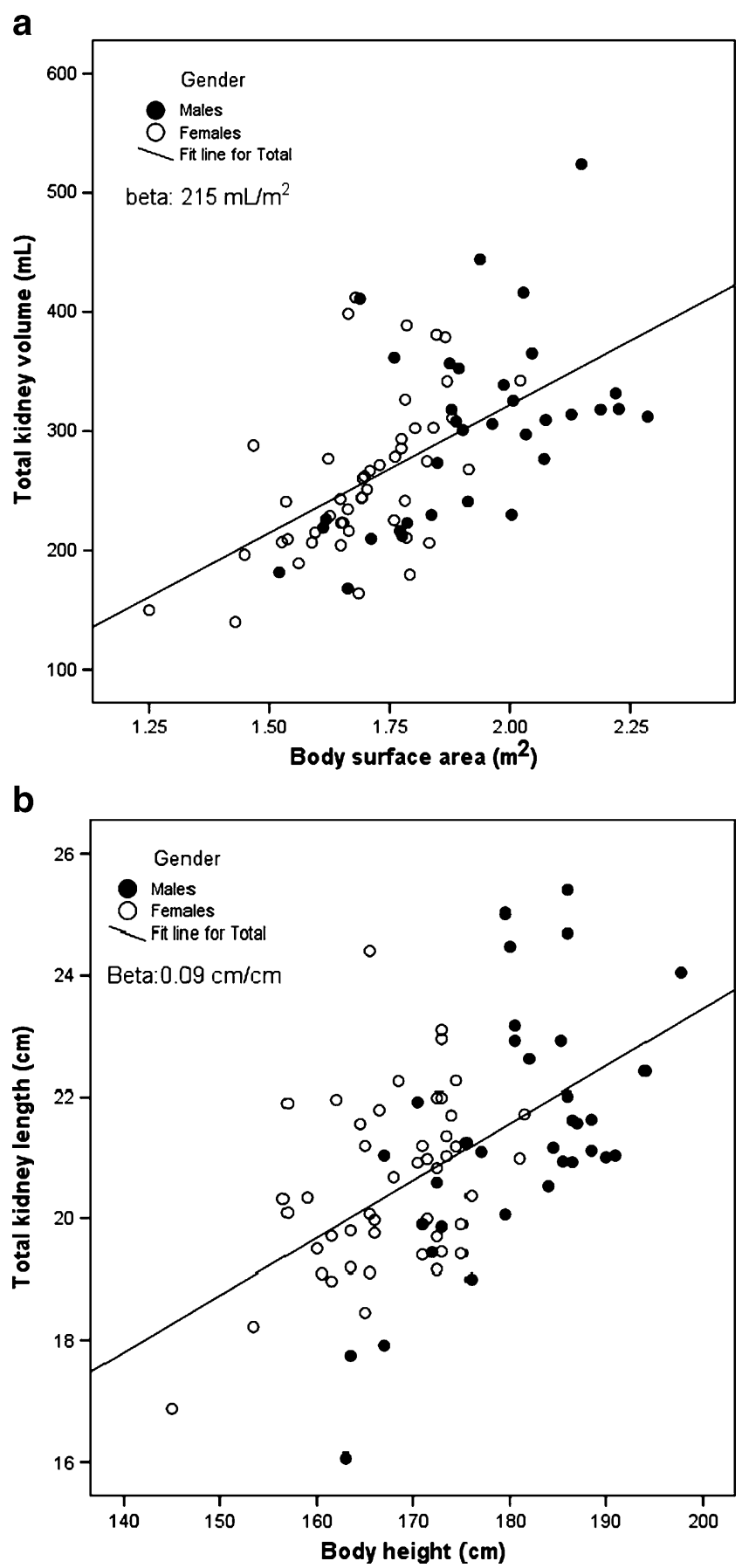

Fig. 3 Relation between body surface area and total kidney volume (a) and body height and total kidney length (b)

height are plotted separately for the left and right kidney against kidney volume and length, respectively.

Renal and ureterovesical anomalies

Renal ultrasound revealed subclinical anomalies of the kidneys and ureters in eight of 51 individuals born prematurely (Table 3 ) and not in controls (Fisher exact test 

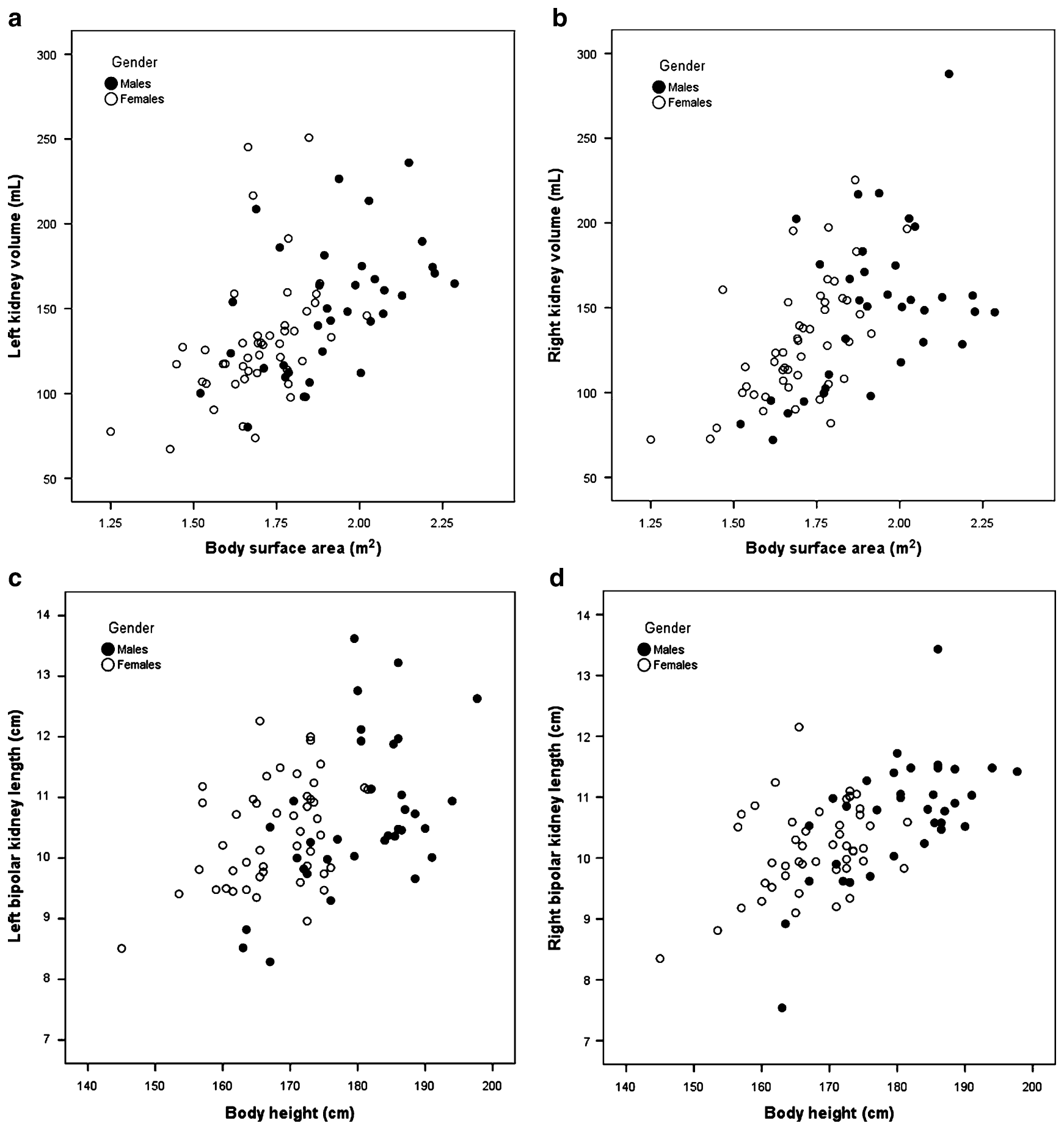

Fig. 4 Body surface area $\left(\mathrm{m}^{2}\right)$ plotted to left (a) and right (b) kidney volume. Body height $(\mathrm{cm})$ plotted to left (c) and right (d) bipolar kidney length

$p$ value $=0.02)$. Anomalies varied from nephrocalcinosis $(N=1)$, unilateral pyelocaliceal dilatation $(N=3)$, ureteropelvic junction obstruction $(N=1)$, ureter dilatation $(N=1)$, extrarenal pelvis $(N=1)$, and ureter duplication and ureterocele $(N=1)$. These were not known before the ultrasound study. Prevalences of the anomalies did not differ between SGA (3/22) and AGA (5/29) individuals. Analyses excluding these eight individuals did not affect findings.

\section{Discussion}

Renal ultrasound study revealed significantly smaller renal length and volume in 20-year-old female individuals born very prematurely, either SGA or AGA, compared with agematched controls. The difference was most pronounced for the left kidney. Differences were observed for male individuals also, but statistical significance was reached 
Table 3 Characteristics of patients with renal anomalies

\begin{tabular}{lllll}
\hline Gender & SGA or AGA & Renal anomaly & Left or right side & GFR $\left(\mathrm{ml} / \mathrm{min} / 1.73 \mathrm{~m}^{2}\right)$ \\
\hline Female & SGA & Unilateral pyelocaliceal dilatation & Right & 118 \\
Female & SGA & Ureter dilatation & Left & 95 \\
Female & SGA & Ureteropelvic junction obstruction & Right & 119 \\
Female & AGA & Pyelocaliceal dilatation & Left and right & 115 \\
Female & AGA & Ureterocele and double ureter & Left & 104 \\
Male & AGA & Nephrocalcinosis & Left and right & 119 \\
Male & AGA & Unilateral pyelocaliceal dilatation & Right & 111 \\
Male & AGA & Extrarenal pelvis & Left and right & 127 \\
\hline
\end{tabular}

$S G A$ small for gestational age prematurely born individuals, $A G A$ appropriate for gestational age prematurely born individuals, $G F R$ glomerular filtration rate

only in female individuals. IUGR had no significant effect on renal size. These results are consistent with impaired fetal kidney development after preterm birth, with probably no more than a small effect of IUGR on renal size. Nephrogenesis starts in early pregnancy, but it peaks at the 32nd week of gestation, continues until 36 weeks of gestation, and is correlated to renal size [5]. The AGA and SGA groups, both born before a gestational age of 32 weeks, did not differ in renal size, possibly because nephrogenesis was not complete at birth. We found differences in renal size between male and female individuals in both the SGA and AGA groups. Such gender differences have only been reported in an experimental IUGR rat model, suggestive of the importance of gender in outcomes in adulthood after IUGR [17]. In our study, differences in renal size between male SGA, AGA, and control individuals seemed smaller than those between female study participants but still showed a trend for decreased absolute renal length and volume. The lack of significance might be due to insufficient power of the study.

Singh et al. described that lower renal volume in Australian Aborigines represents kidneys with reduced nephron number [5]. As there is a limited postnatal nephrogenesis in preterm individuals $[9,10]$, a nephron deficit after preterm birth probably persists throughout life. From a study in 56 deceased extremely premature infants and ten deceased full-term controls, it appeared that nephron number was highly correlated to gestational age and that nephrogenesis had stopped 40 days postnatally [9]. As nephron deficit predisposes to reduced renal function in the adult $[5,6]$, preterm individuals are at such risk. Earlier, we found higher blood pressure and poor renal function and microalbuminuria in these premature individuals $[14,18]$. Furthermore, restricted postnatal growth after premature birth may impair renal function over time [19].

Decreased renal volume was also found in 33 low-birthweight (LBW) Aboriginal children between 5-18 years of age [3]. Their BSA-adjusted renal volume was $78.5 \mathrm{ml} / \mathrm{m}^{2}$ compared with $85.7 \mathrm{ml} / \mathrm{m}^{2}$ in 141 normal birth weight (NBW) individuals $(p=0.018)$. Renal length was equal. The authors stated that LBW resulted from IUGR, but data on gestational age were unavailable in many participants. For that matter, our study found that IUGR did not affect renal size. Renal size differences between preterms and controls were most pronounced for the left kidney, in line with findings in Aboriginal children [3]. Interestingly, hypertensive adults with chronic kidney failure showed smaller absolute left kidney volume (but not right kidney volume) compared with normotensive chronic kidney failure patients [20]. The authors did not study whether renal size in these hypertensive patients was associated with birth weight and gestational age.

Thus, from results of other studies and this study, it would seem that very preterm individuals show limited early postnatal renal growth, especially of the left kidney. Other studies reported that postnatal renal growth after preterm birth was limited until 18-24 months of age but did not mention differences in left and right kidney growth [11, 12]. To our knowledge, only one study described normal fetal kidney growth separately for the left and right kidney [21]. Sonography at several gestational ages did not reveal significant difference between left and right kidney growth between 16 and 38 weeks of gestation. We can only speculate that the left kidney grows more rapidly than the right kidney after the gestational age of 32 weeks in normal gestation, but large studies are needed to confirm this speculation.

Ultrasonography revealed renal anomalies in eight very preterm participants versus none of the controls. The anomalies mainly comprised ectasia or dilatation of the ureters and pyelocaliceal system. All individuals were asymptomatic. It is not known whether the anomalies were already present at birth. They may have developed in the early postnatal period or in childhood but then remained unnoticed, as no ultrasound studies were performed. Some renal diseases, such as acute tubular necrosis and neph- 
rocalcinosis, occur more frequently in prematurely born children, but higher incidences of renal anomalies we found have not yet been reported [22, 23]. One possible biological pathway may be inferred from the observation that in-utero disturbance of the renin-angiotensin system affects normal kidney development [24, 25].

The strength of our study lies in evaluating the effect of prematurity separately from that of IUGR. The difference in renal size was most pronounced between the AGA preterm and full-term participants. The additional effect of IUGR in these very preterm subjects was small and not significant for all renal sizes. Therefore, we suggest that limited renal growth after very preterm birth predisposes to decreased renal size at adult age.

Two possible limitations of the study should be mentioned. First, selection bias may have been introduced by selecting students only as controls. Second, three different radiologists obtained the data of renal size only once. However, to minimize the variability between the three radiologists, renal measurements were made by a standardized protocol, and all radiologists measured subjects from all three groups. Inter- and intraobserver variability testing showed a slight mean error in measurement, which was comparable with other studies $[26,27]$. Moreover, renal lengths measured in controls were similar to those reported by Miletic et al. [28], indicating the radiologists in our study produced accurate measurements.

In conclusion, kidney development after preterm birth is probably stunted, leading to (relatively) smaller kidneys and a higher frequency of renal structural anomalies at the age of 20 years. There is only a small, nonsignificant, additional effect of IUGR on these observations. The effects of stunted development were more obvious in left kidneys and in female individuals. Detailed measurement of renal development, in both size and histological changes, in a large epidemiological study design is required to elucidate the pathophysiological mechanism of unilaterally decreased renal growth after very preterm birth.

Acknowledgements This part of the POPS 19 study was supported by a grant of the Dutch Kidney Foundation. We thank TNO, Quality of Life (ETM Hille, P Verloove-Vanhorick) for the use of the POPS cohort database, MH Lequin for renal ultrasound measurements (Dept. Pediatric Radiology, Erasmus MC - Sophia, Rotterdam), and HA Kleinveld for her efforts in the data collection and analyses (Dept. Pediatric Nephrology, Erasmus MC - Sophia, Rotterdam).

Financial support: Dutch Kidney Foundation, Grant C-1924

No conflict of Interest

Open Access This article is distributed under the terms of the Creative Commons Attribution Noncommercial License which permits any noncommercial use, distribution, and reproduction in any medium, provided the original author(s) and source are credited.

\section{References}

1. Ingelfinger JR (2004) Pathogenesis of perinatal programming. Curr Opin Nephrol Hypertens 13:459-464

2. Woods LL, Weeks DA, Rasch R (2004) Programming of adult blood pressure by maternal protein restriction: role of nephrogenesis. Kidney Int 65:1339-1348

3. Spencer J, Wang Z, Hoy W (2001) Low birth weight and reduced renal volume in Aboriginal children. Am J Kidney Dis 37:915920

4. Konje JC, Okaro CI, Bell SC, de Chazal R, Taylor DJ (1997) A cross-sectional study of changes in fetal renal size with gestation in appropriate- and small-for-gestational-age fetuses. Ultrasound Obstet Gynecol 10:22-26

5. Singh GR, Hoy WE (2004) Kidney volume, blood pressure, and albuminuria: findings in an Australian Aboriginal community. Am J Kidney Dis 43:254-259

6. Hughson M, Farris AB, Douglas-Denton R, Hoy WE, Bertram JF (2003) Glomerular number and size in autopsy kidneys: the relationship to birth weight. Kidney Int 63:2113-2122

7. Brenner BM, Chertow GM (1993) Congenital oligonephropathy: an inborn cause of adult hypertension and progressive renal injury? Curr Opin Nephrol Hypertens 2:691-695

8. Hinchliffe SA, Sargent PH, Howard CV, Chan YF, van Velzen D (1991) Human intrauterine renal growth expressed in absolute number of glomeruli assessed by the disector method and Cavalieri principle. Lab Invest 64:777-784

9. Rodriguez MM, Gomez AH, Abitbol CL, Chandar JJ, Duara S, Zilleruelo GE (2004) Histomorphometric analysis of postnatal glomerulogenesis in extremely preterm infants. Pediatr Dev Pathol $7: 17-25$

10. Rodriguez MM, Gomez A, Abitbol C, Chandar J, Montane B, Zilleruelo G (2005) Comparative renal histomorphometry: a case study of oligonephropathy of prematurity. Pediatr Nephrol 20:945-949

11. Schmidt IM, Chellakooty M, Boisen KA, Damgaard IN, Mau KC, Olgaard K, Main KM (2005) Impaired kidney growth in lowbirth-weight children: distinct effects of maturity and weight for gestational age. Kidney Int 68:731-740

12. Drougia A, Giapros V, Hotoura E, Papadopoulou F, Argyropoulou M, Andronikou S (2009) The effects of gestational age and growth restriction on compensatory kidney growth. Nephrol Dial Transplant 24:142-148

13. Keijzer-Veen MG, Kleinveld HA, Lequin MH, Dekker FW, Nauta J, de Rijke YB, van der Heijden BJ (2007) Renal function and size at young adult age after intrauterine growth restriction and very premature birth. Am J Kidney Dis 50:542-551

14. Keijzer-Veen MG, Finken MJ, Nauta J, Dekker FW, Hille ET, Frolich M, Wit JM, van der Heijden AJ (2005) Is blood pressure increased 19 years after intrauterine growth restriction and preterm birth? A prospective follow-up study in The Netherlands. Pediatrics 116:725-731

15. Schmidt IM, Main KM, Damgaard IN, Mau C, Haavisto AM, Chellakooty M, Boisen KA, Petersen JH, Scheike T, Olgaard K (2004) Kidney growth in 717 healthy children aged $0-18$ months: a longitudinal cohort study. Pediatr Nephrol 19:992-1003

16. Emamian SA, Nielsen MB, Pedersen JF, Ytte L (1993) Kidney dimensions at sonography: correlation with age, sex, and habitus in 665 adult volunteers. AJR Am J Roentgenol 160:83-86

17. Battista MC, Oligny LL, St Louis J, Brochu M (2002) Intrauterine growth restriction in rats is associated with hypertension and renal dysfunction in adulthood. Am J Physiol Endocrinol Metabol 283: E124-E131

18. Keijzer-Veen MG, Schrevel M, Finken MJ, Dekker FW, Nauta J, Hille ET, Frolich M, van der Heijden BJ (2005) Microalbuminuria 
and lower glomerular filtration rate at young adult age in subjects born very premature and after intrauterine growth retardation. J Am Soc Nephrol 16:2762-2768

19. Bacchetta J, Harambat J, Dubourg L, Guy B, Liutkus A, Canterino I, Kassai B, Putet G, Cochat P (2009) Both extrauterine and intrauterine growth restriction impair renal function in children born very preterm. Kidney Int. doi:10.1038/ki.2009.201

20. Zumrutdal AO, Turan C, Cetin F, Adanali S (2002) Relationship between renal size and hypertension in patients with chronic renal failure. Nephron 90:145-147

21. Kennedy WA, Chitkara U, Abidari JM, Shortliffe LM (2003) Fetal renal growth as assessed through renal parenchymal area derived from prenatal and perinatal ultrasonography. J Urol 169:298-302

22. Alexander GR, Himes JH, Kaufman RB, Mor J, Kogan M (2003) A United States national reference for fetal growth. Obstet Gynecol 87:163-168

23. Schell-Feith EA, Que I, Kok DJ, Kist-Van Holthe JE, Kuhler E, Brand R, Papapoulos SE, van der Heijden BJ (2001) Modulation of calcium oxalate monohydrate crystallization kinetics by urine of preterm neonates. Am J Kidney Dis 38:1229-1234
24. Friberg P, Sundelin B, Bohman SO, Bobik A, Nilsson H, Wickman A, Gustafsson H, Petersen J, Adams MA (1994) Renin-angiotensin system in neonatal rats: induction of a renal abnormality in response to ACE inhibition or angiotensin II antagonism. Kidney Int 45:485-492

25. Tufro-McReddie A, Romano LM, Harris JM, Ferder L, Gomez RA (1995) Angiotensin II regulates nephrogenesis and renal vascular development. Am J Physiol 269:F110-F115

26. Ablett MJ, Coulthard A, Lee RE, Richardson DL, Bellas T, Owen JP, Keir MJ, Butler TJ (1995) How reliable are ultrasound measurements of renal length in adults? Br J Radiol 68:10871089

27. Emamian SA, Nielsen MB, Pedersen JF (1995) Intraobserver and interobserver variations in sonographic measurements of kidney size in adult volunteers. A comparison of linear measurements and volumetric estimates. Acta Radiol 36:399401

28. Miletic D, Fuckar Z, Sustic A, Mozetic V, Stimac D, Zauhar G (1998) Sonographic measurement of absolute and relative renal length in adults. J Clin Ultrasound 26:185-189 